\title{
Synthetic JHK Colors for M Dwarfs, M Giants, and Carbon Stars
}

\section{UFFE GRÅE JØRGENSEN ${ }^{1}$ and ROBERT F. WING ${ }^{2}$}

${ }^{1}$ Niels Bohr Institute, University Observatory, Copenhagen, Denmark

2 Ohio State University, Columbus OH, U.S.A.

Using model atmospheres computed with the MARCS code and filter functions for the near-infrared $J, H$, and $K$ filters from Bessell \& Brett (PASP, $100,1134,1988)$, we have computed synthetic $J-H$ and $H-K$ colors for giants $(\log g=0)$ and dwarfs $(\log g=+5)$ of effective temperature $3000-5000 \mathrm{~K}$, both for solar composition and for a carbon-enriched composition $(\mathrm{C} / \mathrm{O}=$ 1.07). The three molecules which have the greatest effects on near-infrared spectra - $\mathrm{CO}, \mathrm{CN}$, and $\mathrm{H}_{2} \mathrm{O}$ - were individualy turned on and off when computing the synthetic spectra in order to investigate their separate effects.

For solar-composition models, we reproduce the observed bifurcation of the giant and dwarf sequences in the $J-H, H-K$ color-color diagram. Absorption by $\mathrm{H}_{2} \mathrm{O}$ has an important effect on the colors of dwarfs, starting at temperatures as high as $4000 \mathrm{~K}$; CO has a noticeable effect on giant colors. However, a major portion (more than half) of the observed bifurcation is present in the model sequences even when no molecules at all are included in the synthetic spectra; this effect is attributed to the differing effects of $\mathrm{H}^{-}$opacity on models having different temperature structures.

In carbon-rich giants the combined effect of $\mathrm{CO}$ and $\mathrm{C}_{2}$ is to change the $J-H$ and $H-K$ colors considerably from the corresponding continuum colors. For the coolest models $\mathrm{C}_{2} \mathrm{H}_{2}$ and $\mathrm{C}_{3}$ have a strong effect in the $K$ filter. In carbon dwarfs $\mathrm{CO}$ and $\mathrm{C}_{2}$ have small and opposite effects on the $J \mathrm{HK}$ colors, and the colors of the dwarfs are therefore similar to those of the continuum alone. 